\title{
Asymptomatic Pulmonary Cement Embolism after Cement-Augmented Pedicle Screw Instrumentation: A Case Report
}

\author{
Hyun Jun Kim${ }^{1}$, Seok Won Chung ${ }^{1}$, Sang Ho Lee ${ }^{2}$, Chan Hong Park ${ }^{3}$, Yong Hwan Shin ${ }^{1}$, \\ Shin Young Lee ${ }^{1}$, Min Soo Kang ${ }^{1}$ \\ ${ }^{1}$ Department of Neurosurgery, Daegu Wooridul Spine Hospital, Daegu, \\ ${ }^{2}$ Department of Neurosurgery, Wooridul Spine Hospiral, Seoul, \\ ${ }^{3}$ Department of Anesthesiology and Pain Medicine, Daegu Wooridul Spine Hospital, Daegu, Republic of Korea
}

Corresponding author:

Seok Won Chung

Department of Neurosurgery,

Daegu Wooridul Spine Hospital,

648, Gukchaebosang-ro, Jung-gu,

Daegu 41939, Republic of Korea

Tel: +82-53-212-3000

Fax: +82-53-212-3049

E-mail: neurochung79@gmail.com

Received: January 20, 2020

Revised: March 3, 2020

Accepted: March 9, 2020
Cement-augmented pedicle screw instrumentation (CAPSI) is a widely used surgical technique in osteoporotic patients. Since CAPSI improves the pull-out strength of screws and enhances bone density of the screwed vertebral body, it can prevent fixation related complications such as screw loosening, and cage subsidence can be prevented. However, several complications due to cement leakage have been reported. Herein we describe a rare case of asymptomatic pulmonary cement embolism (PCE) after CAPSI. A 65-year-old osteoporotic patient was diagnosed with Kummell's disease at T10. Vertebroplasty at T10 and cement augmented posterior fixation with posterolateral fusion were performed at 2 levels above and below. Postoperatively mild fever, and sputum formation were observed. On a simple chest tomographic image, diffuse PCE was visible on both lung parenchyma. Since his symptoms were not related to PCE no anticoagulant was added to his medication and he was managed conservatively. After conservative care, he was discharged without pulmonary symptom. PCE after CAPSI has been reported in several articles and it can end in the death of the patient. When asymptomatic PCE is detected, close observation with conservative care is a feasible therapeutic strategy.

Key Words: Bone cements; Pedicle screws; Pulmonary embolism

\section{INTRODUCTION}

Pedicle screw fixation is widely performed for the treatment of patients suffering from trauma, tumors, and degenerative diseases. As the proportion of elderly individuals in the population increases, the demand for surgical management for osteoporotic patients with poor bone quality has also increased. Screws insertion in osteoporotic vertebrae became a prevalent challenge. Pedicle screwing in the osteoporotic cancellous bone can result in screw loosening, or bone collapse. To overcome this issue, several surgical techniques and instruments were developed such as bicortical purchase of the screw, expandable screws, and cement-augmentation pedicle screw instrumentation (CAPSI). Cement augmentation not only provides an optimal fixation strength, but also enhances the density of the instrumented vertebral body, leading to prevent further subsidence of the vertebral body and the progression of kyphotic deformity. The biomechanical efficacy of CAPSI with poly- methylmethacrylate (PMMA) and calcium ceramics has been demonstrated in many articles"). CAPSI has been found to enhance pull-out strength in both osteoporotic vertebrae and revision spine surgery models. However, there are several complications related to cement insertion such as cement leakage through the venous system or adjacent bone fractures. Local cement leakage to the spinal cord or nerve root through the basivertebral veins can cause myelopathy or radiculopathy. Unlike the complications from cement leakage into the basivertebral veins, leakage into segmental veins was reported to be related to more systemic and severe complications like pulmonary cement embolism (PCE) ${ }^{9,17)}$. If the embolic volume of the cement is not large enough to block the main vessels of the heart or lung, the leak is unlikely to cause adverse symptoms. However, when the volume is large enough to interfere with venous flow, the result can be fatal. Herein we report a rare case of asymptomatic PCE after CAPSI in a severely osteoporotic patient. We present the following case in accordance with the CARE Guideline. 


\section{CASE REPORT}

A 65-years-old man visited the outpatient clinic due to persistent severe back pain with a pain intensity of 8 on the visual analog scale (VAS) and worsening paraparesis over a period of 2 months. He was diagnosed with T10 burst fracture 2 month earlier at a private clinic. After 2 weeks of bed rest, self-ambulation was possible with stabilizing orthosis and he was treated conservatively. However, his pain later became aggravated and motor power for both lower limbs weakened

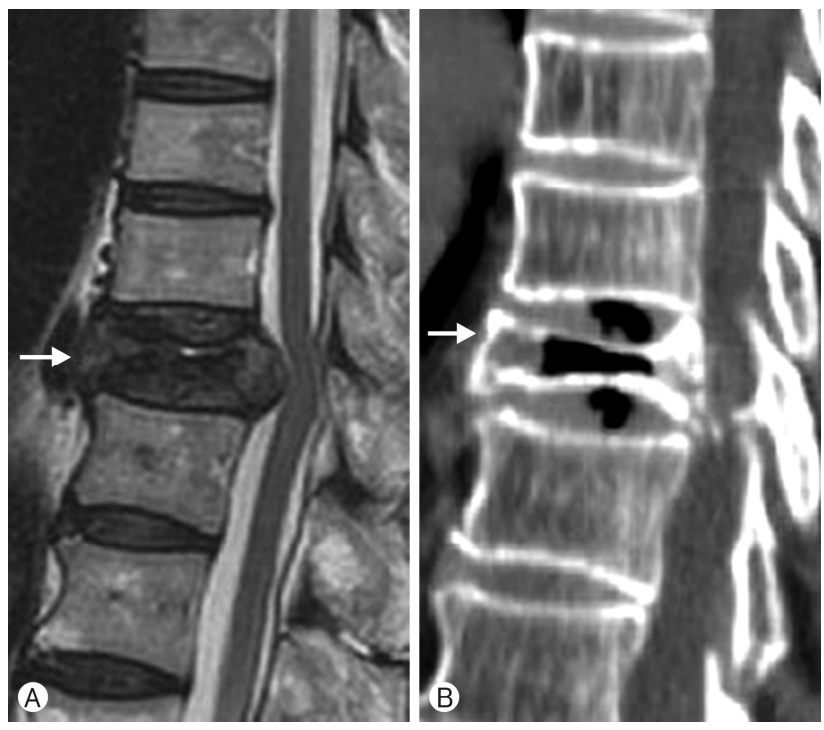

Fig. 1. (A) T2 sequence sagittal magnetic resonance image shows high signal change on spinal cord due to encroachment of fractured bony fragment at T10 level (white arrow). (B) Computed tomography shows air density at intervertebral space of $\mathrm{T} 10$ vertebral body (white arrow).
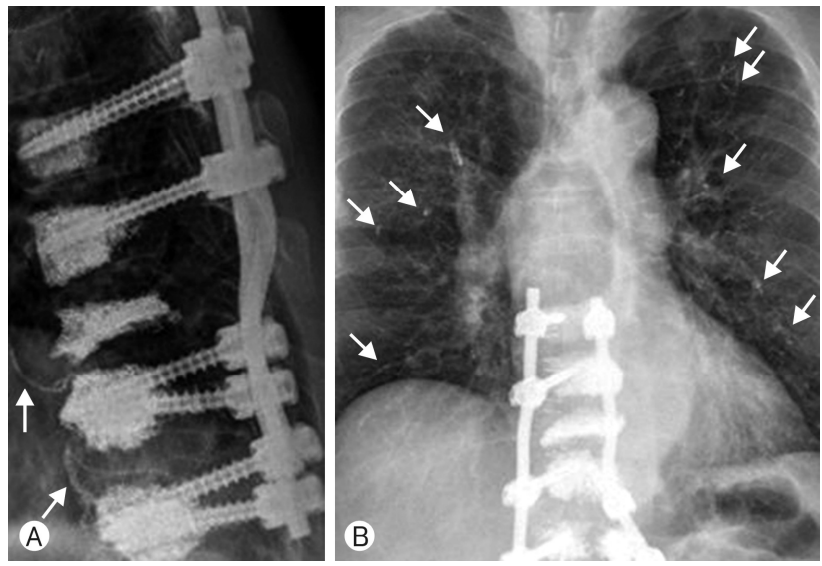

Fig. 2. (A) Postoperative thoracolumbar simple tomograph shows cement leakage at anterior segmental vein of $\mathrm{T} 11, \mathrm{~T} 12$ (white arrows). (B) Chest X-ray shows multiple hyperdense lesions on both lung parenchyma indicating pulmonary cement embolism (white arrows). to grade 3 , causing him to visit to the outpatient clinic in a wheel chair.

On magnetic resonance image and computed tomography (CT), partial bony encroachment and spinal cord compression at the T10 level were visible (Fig. 1). We diagnosed him with Kummell's disease. Severe osteoporosis was confirmed by a T-score of -3.3 on dual-energy X-ray absorptiometry (GE Lunar, Madison, WI, USA) scan. Vertebroplasty (VP) at T10 and cementaugmented posterior fixation with posterolateral fusion 2 levels above and below were planned. After VP was performed with 6 cc of PMMA through bilateral pedicles on T10, each pedicle screw insertion site was augmented with 2 cc of PMMA. During the injection of cement at T11 and 12 cement leakage to the anterior segmental veins was visible. Further injection was immediately halted and there was no obvious unstable hemodynamic change.

Postoperative thoracolumbar simple tomography showed cement leakage at the anterior segmental vein of T11, T12 and chest X-ray showed multiple PCE on both lung parenchyma (Fig. 2). Since the patient experienced no respiratory distress or chest pain he was treated conservatively with mucolytics and oral analgesia. On post-operative day (POD) 2, his back pain subsided significantly (VAS 3) and he could walk with the assistance of a walker. Line 76: POD 6, the patient had mild fever and sputum with an increased level of C-reactive protein (CRP; $249 \mathrm{mg} / \mathrm{L})$. Therefore, intravenous $2^{\text {nd }}$ generation cephalosporin was administered. There were still no signs of dyspnea, tachypnea or cyanosis and arterial blood gas analysis was within the normal range. Since his symptoms were not related to PCE we didn't add any anticoagulant to his medication. On POD 16 his muscle strength of lower extremity improved to grade 4 and he could walk without assistance. CRP levels had decreased to $9.8 \mathrm{mg} / \mathrm{L}$ and he was discharged without any pulmonary symptoms.

\section{DISCUSSION}

The incidence of PCE in VP or kyphoplasty (KP) was reported to be $3.5 \%$ to $23 \%^{10,11)}$. Although the incidence of PCE after CAPSI is not well known, there have been several case reports and clinical studies reporting the occurrence of PCE following the procedure. Those were found on pubmed.org by searching with keywords of "cement", "pedicle screw", and "embolism" and symptomatic cases are listed in Table 1. There were total 103 cases of PCE after CAPSI reported in the literature and 34 of these patient suffered from symptoms such as shortness of breath, dyspnea, tachypnea, cyanosis, chest pain, cough, hemoptysis, dizziness, and sweating. The other patients were asymptomatic. Unfortunately 3 patients out of 34 were died due to fulminant pulmonary embolism and one patient underwent pulmonary arteriotomy ${ }^{6}$. For patients who experienced mild or no symptoms, close observation with conservative care or prophylactic anticoagulation was undertaken. PCE was 
Table 1. Reported PCE after CAPSI (articles are listed based on published year)

\begin{tabular}{|c|c|c|c|c|c|}
\hline References & Year & $\begin{array}{c}\text { Type of screw and } \\
\text { augmentation material }\end{array}$ & $\begin{array}{l}\text { No. of PCE cases } \\
\text { (PCE/total) }\end{array}$ & $\begin{array}{c}\text { Symptom } \\
\text { (symptomatic patients/PCE) }\end{array}$ & Treatment \\
\hline Rasch et al. ${ }^{11)}$ & 2010 & Solid/Palacos & $1 / 1$ & Dyspnea, hypoxia & Pulmonary arteriotomy \\
\hline Tonolini and Bianco ${ }^{14)}$ & 2012 & Solid & $1 / 1$ & Dyspnea, tachypnea & LMWH and warfarin \\
\hline El Saman et al. ${ }^{4)}$ & 2013 & Fenestrated & $12 / 42$ & No life-threatening symptoms & - \\
\hline Zheng et ll. $^{20)}$ & 2013 & Solid & $1 / 1$ & Sudden death & - \\
\hline Erdem et al. ${ }^{6)}$ & 2017 & Solid & $8 / 31$ & Chest discomfort (8/8) & LMMH and warfarin \\
\hline Janssen et al. ${ }^{91}$ & 2017 & Solid & $13 / 165$ & 3 deaths, 2 anaphylactic shock (5/13) & LMMH and warfarin \\
\hline Ulusoy et al. ${ }^{16)}$ & 2018 & Fenestrated & $46 / 281$ & Hemodynamic instability $(4 / 46)$ & LMWH and warfarin \\
\hline Guo et al. ${ }^{7}$ & 2019 & Solid & $11 / 202$ & Dyspnea, instability (2/1 1) & Conservative care \\
\hline
\end{tabular}

PCE: pulmonary cement embolism; CAPSI: cement-augmented pedicle screw instrumentation; LMWH: low-molecular-weight heparin.

occurred with both solid and fenestrated pedicle screws, but Sun et $a{ }^{13)}$ reported that cement leakage was significantly less common in the fenestrated screw group. The incidence of PCE may be underestimated because postoperative chest CT scans are rarely performed and most patients are asymptomatic as in our case. Some cases presented an insidious onset several months after surgery, therefore thorough evaluation of postoperative simple chest X-ray images is essential.

Cement leakage can occur in several types of peri- or intravertebral body veins. These include the basivertebral veins, hemiazygos veins, azygos veins and segmental veins. The segmental veins are known to connect directly to major vessels like azygos vein or inferior vena cava (IVC) which connect to the right heart and pulmonary artery"). Above the T10 vertebral levels some azygos or hemiazygos veins directly drains into the pulmonary artery ${ }^{16}$. Therefore cement leakage to the perivertebral segmental, hemiazygos or azygos veins should be considered as main route to PCE. These 2 types of vein are relatively short and tend to connect directly to the main vessels. Cement leakage through the segmental vein was correlated with right-side-pedicles and a large volume of cement in a previous study ${ }^{7}$. When a large volume of cement occupies a small cavity, the intracavitary pressure increases, causing extravasation of the cement to the segmental veins. Right segmental veins are closer to the IVC and shorter than veins on the other side.

A larger volume of cement, lower viscosity, higher fracture severity grade, the presence of an intravertebral cleft, and the lack a cavity creation prior to cement insertion are risk factors for cement leakage ${ }^{7)}$. The risk factors of cement leakage to these vessels can be divided into 3 groups. Risk factors in the first group are related to poor bone quality. In osteoporotic patients, cancellous bone of the vertebral body is more porous and thus has a higher probability of leakage. Malignant tumor on vertebral body defects the cortical bone and neo vascularization are reasons for more cement leakage ${ }^{8)}$. In trauma patients, higher fracture severity grade, and the presence of an intravertebral cleft are the risk factors. Risk factors in the second group are related the level or number of operated vertebrae.
Instrumentation for more than six segments and thoracic level surgery are considered riskier. Proximity to the pulmonary veins and heart would be a reason for frequent PCEs following thoracic level CAPSI"). Risk factors in the third group are related to surgical technique, and instruments. Viscosity and the amount of cement material used are critical factors. Viscosity is not a measurable value; therefore, despite the ambiguity, toothpaste-like viscosity is generally recommended. Low viscosity can cause early leakage before the hardening of the cement by polymerization of the PMMA. The higher the volume of cement used the higher the risk of cement leakage will be, as reported in several articles. Too much cement will increase pressure in the intra-vertebral body cavity, facilitating the migration of cement to the IVC ${ }^{2,15)}$. Creating a large enough cavity prior to cement augmentation is recommended to control the intracavitary pressure. This is supported by the findings from a post-mortem cohort in which PCE was significantly more common in the VP group than the KP group ${ }^{3)}$. Technically, gradually pulling out the bone needle while injecting the cement will result in a larger cavity and help adjust the intracavitary pressure. In our case, Even though the patient had inevitable risk factors like severe osteoporosis, and multiple thoracic instrumented levels, PCE could be prevented by recognizing the importance of the technical and material-related risk factors and concentrating on controlling them.

Close observation and conservative care would be sufficient for asymptomatic patients. Long-term follow-up of asymptomatic patients without preventive anticoagulation resulted in no symptom development ${ }^{3)}$. However, insidious onset symptoms from PCE were reported in several case reports; therefore, awareness of the treatment options for symptomatic PCE is necessary ${ }^{19)}$. Even though the effect of preventive anticoagulation has not been well established, intravenous heparin followed by oral anticoagulants has been used to manage PCE patients ${ }^{10)}$. This approach is believed to prevent thrombus formation on the embolized cement ${ }^{3)}$. Although the regimen for anticoagulation and antiplatelets varies, intravenous heparinization followed by six months of coumarin therapy was recommend by some authors ${ }^{10)}$. If the patient is unstable hemo- 
dynamically more invasive treatment should be considered. Small linear or string-like and bendable fragments are more suitable for endovascular extraction ${ }^{19)}$. Even when leaked cement settles in the segmental vein, it can migrate later to the IVC or pulmonary artery. To prevent further migration these fragments can be retrieved preemptively using endovascular snares. A surgical approach should be considered when the cement pieces are too large to be extracted thorough the femoral catheter window $^{19)}$. A cement embolus at the main trunks of the pulmonary arteries it can induce hemodynamic instability. Tozzi et al. ${ }^{15)}$ reported such a case, which was treated with open pulmonary embolectomy after CAPSI. Krueger et al. ${ }^{10)}$ reported 5 cases that required open cardiac surgery to remove cement from the central pulmonary artery and right ventricle after VP. Even diffuse peripheral cement emboli can cause lobar necrosis which requires surgical resection ${ }^{12)}$.

No treatment is superior to prevention; thus, there are several suggestions for the prevention of PCE. These can be categorized into 3 groups. The first is to prevent leakage by thorough fluoroscopic inspection during the procedure. To do so, more vivid visualization of cement leakage is required by increasing the proportion of barium in PMMA, which can help with early detection. Once cement extravasation to the segmental vein is detected the procedure should be halted, because the vein is directly connected to the azygos vein and IVC. Leakage through the segmental veins was detected in 14\% of lateral radiographs and $52 \%$ of anterior-posterior (AP) views ${ }^{17)}$. Since the lateral view is mainly utilize during CAPSI, frequent verification of leakage with the AP view is recommended. Concurrent venography can also be considered before cement injection for selective use embolization agent. However the use of contrast media for venography may interfere with early detection of cement leakage, and is therefore controversial ${ }^{8)}$.

The second suggestion is controlling the intracavitary pressure through cement material or surgical technique. A comparative study between cement-augmented traditional pedicle screws and fenestrated screws showed similar clinical and perioperative outcomes, but cement leakage was significantly less common in the fenestrated screw group ${ }^{13)}$. On the other hand when fenestrated screws are used extra attention at location of proximal fenestrations is necessary to prevent posterior leakage ${ }^{5}$. In terms of cement leakage, PMMA is better than ceramic bone cements, because ceramic cements have lower viscosity $^{5,18)}$. A proper toothpaste-like viscosity is ideal, as liquefied PMMA can leak before polymerization. Injecting highly viscous cement leads to the polymerization of PMMA in a short time, reducing the spreading distance which can lead to insufficient cement injection. Zhang et al. ${ }^{18)}$ reported in their comparative study that significant cement leakage was observed in a randomly designated high viscosity group. The optimal mount of cement should be enough to form a strong bonescrew interface, but not enough to induce extravasation of the cement to the neighboring venous plexus. The injection of $1.0 \mathrm{cc}$ or $1.5 \mathrm{cc}$ increases the pull out strength to $58.1 \%$. Although the pull out strength can increase with up to $3 \mathrm{cc}$ of cement augmentation, a maximum of $2.6 \mathrm{cc}$ is recommended for osteoporotic patients at the thoracic levels, and $1.0 \mathrm{cc}$ and $1.5 \mathrm{cc}$ provide sufficient axial pullout strength ${ }^{5)}$. With respect to the surgical technique, sequential cementation at each level is recommended"). Staged injection technique with a small amount of cement allows solidification of the previously injected cement which will blocks endangered veins. In addition to this tapping with ballooning of the KP instrument prior to CAPSI will create a cavity large enough to prevent intracavitary pressure increase and seal the venous drainage by compressing the cancellous bone.

Lastly PCE can be prevented by using of preventive material or controlling systemic pressure. Gelfoam preinjection embolization was reported to be an effective technique for preventing cement leakage $^{1)}$. Bhatia et al. ${ }^{1)}$ performed gelfoam embolization prior to cement insertion routinely for osteoporotic patients, and reported 2\% epidural leakage rate. Preoperative placement of IVC filter could also be considered as a preventive step $^{13)}$. El Saman et al. ${ }^{4}$ insisted that a positive end expiratory pressure of $15 \mathrm{cmH}_{2} \mathrm{O}$ could reduce local cement leakage. The underlying mechanism is the temporary induction of high pressure in the IVC and segmental vein, which can suppress pressure in the tapped cavity before cement augmentation ${ }^{7}$.

\section{CONCLUSION}

PCE after CAPSI was reported in several articles and can result in the death of the patient. Anticoagulants, cardiovascular intervention, or surgery should be considered for symptomatic PCE patients. As prevention is the best way to manage PCE, caution regarding the cement viscosity and, amount as well as the control of intracavitary pressure is required. When asymptomatic PCE is detected, close observation with conservative care is a feasible therapeutic strategy.

\section{CONFLICTS OF INTEREST}

No potential conflict of interest relevant to this article was reported.

\section{REFERENCES}

1. Bhatia C, Barzilay Y, Krishna M, Friesem T, Pollock R: Cement leakage in percutaneous vertebroplasty: effect of preinjection gelfoam embolization. Spine (Phila Pa 1976) 31:915-919, 2006

2. Butscheidt S, Rolvien T, Ritter J, Heinemann A, Vogel H, Amling $\mathrm{M}$, et al.: Pulmonary cement embolism is not associated with the cause of death in a post-mortem cohort of cement-augmented interventions in the spine. Eur Spine J 27:2593-2601, 2018 
3. Choe DH, Marom EM, Ahrar K, Truong MT, Madewell JE: Pulmonary embolism of polymethyl methacrylate during percutaneous vertebroplasty and kyphoplasty. AJR Am J Roentgenol 183:1097-1102, 2004

4. El Saman A, Kelm A, Meier S, Sander AL, Eichler K, Marzi I, et al.: Intraoperative PEEP-ventilation during PMMA-injection for augmented pedicle screws: improvement of leakage rate in spinal surgery. Eur J Trauma Emerg Surg 39:461-468, 2013

5. Elder BD, Lo SF, Holmes C, Goodwin CR, Kosztowski TA, Lina IA, et al.: The biomechanics of pedicle screw augmentation with cement. Spine J 15:1432-1445, 2015

6. Erdem MN, Karaca S, Sari S, Yumrukcal F, Tanli R, Aydogan $\mathrm{M}$ : Application of cement on strategic vertebrae in the treatment of the osteoporotic spine. Spine J 17:328-337, 2017

7. Guo HZ, Tang YC, Guo DQ, Zhang SC, Li YX, Mo GY, et al.: The cement leakage in cement-augmented pedicle screw instrumentation in degenerative lumbosacral diseases: a retrospec tive analysis of 202 cases and 950 augmented pedicle screws. Eur Spine J 28:1661-1669, 2019

8. Jang JS, Lee SH, Jung SK: Pulmonary embolism of polymethylmethacrylate after percutaneous vertebroplasty: a report of three cases. Spine (Phila Pa 1976) 27:E416-E418, 2002

9. Janssen I, Ryang YM, Gempt J, Bette S, Gerhardt J, Kirschke JS, et al.: Risk of cement leakage and pulmonary embolism by bone cement-augmented pedicle screw fixation of the thoracolumbar spine. Spine J 17:837-844, 2017

10. Krueger A, Bliemel C, Zettl R, Ruchholtz S: Management of pulmonary cement embolism after percutaneous vertebroplasty and kyphoplasty: a systematic review of the literature. Eur Spine J 18:1257-1265, 2009

11. Rasch A, Sindermann J, Scheld HH, Hoffmeier A: Pulmonary Palacos embolism: a case report. Thorac Cardiovasc Surg 58: 437-439, 2010

12. Rothermich MA, Buchowski JM, Bumpass DB, Patterson GA: Pulmonary cement embolization after vertebroplasty requiring pulmonary wedge resection. Clin Orthop Relat Res 472:1652-
1657,2014

13. Sun HL, Li CD, Yang ZC, Yi XD, Liu H, Lu HL, et al.: Polymethylmethacrylate augmentation of bone cement-injectable cannulated pedicle screws for the treatment of degenerative lumbar diseases with osteoporosis. Beijing Da Xue Xue Bao Yi Xue Ban 48:1019-1025, 2016

14. Tonolini M, Bianco R: Pulmonary cement embolism after pedicle screw vertebral stabilization. J Emerg Trauma Shock 5: 272-273, 2012

15. Tozzi P, Abdelmoumene Y, Corno AF, Gersbach PA, Hoogewoud HM, von Segesser LK: Management of pulmonary embolism during acrylic vertebroplasty. Ann Thorac Surg 74:17061708, 2002

16. Ulusoy OL, Kahraman S, Karalok I, Kaya E, Enercan M, Sever C, et al.: Pulmonary cement embolism following cement-augmented fenestrated pedicle screw fixation in adult spinal deformity patients with severe osteoporosis (analysis of 2978 fenestrated screws). Eur Spine J 27:2348-2356, 2018

17. Yeom JS, Kim WJ, Choy WS, Lee CK, Chang BS, Kang JW: Leakage of cement in percutaneous transpedicular vertebroplasty for painful osteoporotic compression fractures. J Bone Joint Surg Br 85:83-89, 2003

18. Zhang L, Wang J, Feng X, Tao Y, Yang J, Wang Y, et al.: A comparison of high viscosity bone cement and low viscosity bone cement vertebroplasty for severe osteoporotic vertebral compression fractures. Clin Neurol Neurosurg 129:10-16, 2015

19. Zhao Y, Liu T, Zheng Y, Wang L, Hao D: Successful percutaneous retrieval of a large pulmonary cement embolus caused by cement leakage during percutaneous vertebroplasty: case report and literature review. Spine (Phila Pa 1976) 39:E1616E1621, 2014

20. Zheng N, Liang M, Zhang HD, Zhu SH, Yang TT, Zhuo L, et al.: Fatal extensive bone cement embolism: histological findings confirmed by Fourier transform infrared spectroscopy. Forensic Sci Int 229:e23-e25, 2013 